Research paper

\title{
Examination of the risk of reinfection with hepatitis $C$ among injecting drug users who have been tested in Glasgow
}

\author{
Scott A. McDonald ${ }^{\mathrm{a}, \mathrm{b}, *}$, Sharon J. Hutchinson ${ }^{\mathrm{a}, \mathrm{b}}$, Sheila O. Cameron ${ }^{\mathrm{c}}$, Hamish A. Innes ${ }^{\mathrm{a}, \mathrm{b}}$, Allan McLeod ${ }^{\mathrm{a}}$, \\ David J. Goldberga
}

${ }^{a}$ Health Protection Scotland, Meridian Court, 5 Cadogan Street, Glasgow G2 6QE, Scotland, UK

b Department of Mathematics and Statistics, University of Strathclyde, 26 Richmond Street, Glasgow G1 1XH, Scotland, UK

${ }^{\mathrm{c}}$ West of Scotland Specialist Virology Centre, Gartnavel General Hospital, Glasgow, UK

\section{A R T I C L E I N F O}

\section{Article history:}

Received 9 October 2011

Received in revised form 3 February 2012

Accepted 6 February 2012

\section{Keywords:}

Hepatitis C virus

Intravenous substance abuse

Incidence

Epidemiology

\begin{abstract}
A B S T R A C T
Background: Unsafe injecting practices put injecting drug users (IDUs) at repeat exposure to infection with the hepatitis C virus (HCV). It has not yet been determined if spontaneously clearing one's primary infection influences the risk of reinfection; our aim was to estimate the relative risk of reinfection in IDUs who have cleared the virus.

Methods: We conducted a retrospective study using a large database of HCV test results covering Greater Glasgow Health Board during 1993-2007 to calculate rates of infection and reinfection in current/former IDUs. The relative risk of (re)infection in previously infected compared with never-infected IDUs was estimated using Poisson regression, adjusting for age at study entry, sex, and calendar period of test. Results: Although the rate of reinfection in IDUs who were HCV antibody-positive, RNA-negative at baseline was lower (7/100 person-years, 95\% CI: 5-9) than the rate of acute infection in IDUs who were HCV antibody-negative at baseline (10/100 person-years, 95\% CI: 9-12), the risk of reinfection was not significantly different than the risk of initial infection (adjusted rate ratio $=0.78,95 \% \mathrm{CI}$ : $0.57-1.08$ ). Conclusion: We found only weak evidence for a reduced risk of HCV reinfection in IDUs who had cleared their previous infection. Further research among those who have cleared infection through antiviral therapy is needed to help inform decisions regarding treatment of IDUs.
\end{abstract}

(c) 2012 Elsevier B.V. All rights reserved.

\section{Introduction}

The Scottish Government's recent initiative addressing the hepatitis Cepidemic (Scottish Government Health Department, 2008) in which nationwide prevalence among injecting drug users (IDUs) is estimated at $53 \%$ in 2008 , with $1000-1500$ IDUs newly acquiring hepatitis $C$ virus infection (HCV) each year (Scottish Government, 2011) - aimed to increase the number of infected individuals commencing treatment from 500 per year in 2008/2009 to at least 2000 per year from 2011/2012. Data regarding reinfection rates are needed to inform on efforts to prevent HCV among IDUs, as well as the treatment of current IDUs. A clearer understanding of the risks of reinfection and the possibility of protective partial immunity in active IDUs is important when planning the provision of antiviral therapy, particular among individuals with ongoing risk exposure.

Approximately $26 \%$ of persons with acute $\mathrm{HCV}$ infection are able to spontaneously clear the virus (Micallef, Kaldor, \& Dore, 2006) and

\footnotetext{
* Corresponding author at: Health Protection Scotland, Meridian Court, 5 Cadogan Street, Glasgow G2 6QE, Scotland, UK. Tel.: +44 1413001106 ; fax: +441413001170.

E-mail address: smcdonald4@nhs.net (S.A. McDonald).
}

the remainder become chronically infected, with the consequent risk of HCV-related disease progression: liver fibrosis, cirrhosis and hepatocellular carcinoma. For IDUs, who represent the largest at-risk group in Australia, USA, UK and other Western European countries (Alter et al., 1999; Dore, MacDonald, Law, \& Kaldor, 2003; Health Protection Agency, 2009; Trepo \& Pradat, 1999), the majority of transmission occurs through the sharing of contaminated injecting equipment, and so there is much opportunity for reinfection among those IDUs who do not use clean needles/syringes on each injecting occasion.

There is a lack of consistent evidence regarding the question of whether previous HCV infection confers partial immunity upon reexposure, and so leads to a lower risk of re-infection compared with the risk of new infection (Corson, Greenhalgh, Palmateer, Weir, \& Hutchinson, 2011; Currie et al., 2008; van de Laar et al., 2009); such a lower risk of reinfection was observed in three cohort studies (Farci et al., 1992; Grebely et al., 2006; Mehta et al., 2002). However, two other studies indicated a higher infection rate among previously-infected compared with never-infected IDUs (Aitken et al., 2008; Micallef et al., 2007), although the incidence rate ratio in the latter study was not statistically significant after adjustment for behavioural covariates. 
Our goal was therefore to estimate the relative risk of reinfection after viral clearance, by comparing rates of initial infection (in those testing antibody[Ab]-/PCR-) and reinfection (in those testing $\mathrm{Ab}+\mathrm{PCR}-$, that is, who had cleared their primary infection) among IDUs identified within a large laboratory database of HCV test results for Greater Glasgow Health Board (GGHB). An estimated 40\% $(20,000 / 50,000)$ of Scotland's HCV infected population (Scottish Government Health Department, $2008)$ and $37 \%(8862 / 23,933)$ of mainland Scotland's IDU population (Hay, Gannon, Casey, \& McKeganey, 2009) currently live within GGHB.

\section{Methods}

\section{Study population and data sources}

Health Protection Scotland (HPS) maintains a database through sourcing records from the West of Scotland Specialist Virology Centre - of all persons who have undergone an HCV $\mathrm{Ab}$ and/or HCV PCR test in NHS Greater Glasgow Health Board. $\mathrm{HCV}$ antibody reactivity in blood specimens was detected using an enzyme linked immunosorbent assay (ELISA) and a supplemental RIBA-3 test (Ortho Diagnostics). The presence of HCV RNA was ascertained using an in-house method following a positive ELISA result. A second sample is immediately requested from the clinician to confirm RNA status in $\mathrm{HCV}$ Ab reactive individuals; therefore, initial test result was defined as either an $\mathrm{Ab}$ - test, or an initial $\mathrm{Ab}+$ test followed by a PCR test within 1 month.

Individual records on the HCV test database held at HPS contain the following non-named information: sex, date of birth, surname soundex code, and forename initial, as well as data concerning risk activities (classified into reported IDU and not-known). We used a strict deterministic approach (that is, required complete matches on all available identifiers) to map individual test records to distinct persons. Following this step, data for 97,250 individuals who had been tested at least once for HCV between 1993 and 2007 were available. Extra data on risk activities were obtained via deterministic record-linkage to the national HIV test database also held by HPS (Goldberg, Davis, Allardice, McMenamin, \& Codere, 1996). This database contained records for 415,555 HIV tests conducted over the period 1988-2007, among which 36,618 mention IDU as risk activity. 26,807 (27.6\%) of HCV-tested persons linked to the HIV test database.

Two subcohorts were defined: (i) those current/former IDUs who had no evidence of past HCV infection, that is, initial test result was $\mathrm{Ab}$ - and if also PCR tested at the same time, PCR- (the Never-infected subcohort); and (ii) those IDUs with evidence of past infection but no viraemia at initial test, that is, Ab+/PCR- (the previously-infected subcohort).

We checked that our previously-infected subcohort did not include IDUs who had received antiviral therapy, to guard against treatment relapse being interpreted as reinfection. This was done via deterministic record-linkage to the Scottish HCV Clinical Database (a database containing clinical follow-up data on HCV infected patients attending antiviral treatment clinics in Scotland), matching on date of birth, sex, and initials. Only one IDU was identified, who achieved a sustained response and was PCR- at second test.

The study population thus consisted of those individuals on the HCV test database who had complete data on sex and date of birth (and so excluding 17 persons), who had IDU indicated as risk activity leading to infection, who were initially HCV tested in the period 1993-2007, and had at least one subsequent HCV PCR test (or $\mathrm{Ab}-$ if no subsequent PCR test) (Never-infected subcohort; $n=829$ ), or at least one subsequent PCR test (previouslyinfected subcohort; $n=347$ ). Based on linkage to the HIV test database, $10 / 1176$ participants $(<0.1 \%)$ were determined to be HIVinfected.

\section{Data analysis}

We computed frequencies and rates of the incidence of (re-)infection in the two subcohorts defined above. First, in those IDUs who initially tested $\mathrm{Ab}+/ \mathrm{PCR}-$ (previously-infected subcohort), follow-up time was defined as time from initial test to the midpoint of the interval between the date of the last negative PCR test and the date of the first positive PCR test. If no positive PCR test was ever recorded for an individual, follow-up time was censored at date of the last negative PCR test. 'Reinfection' was defined as such if a member of the previously-infected cohort tested PCR+ at least once during follow-up. Second, in those IDUs who tested $\mathrm{Ab}-\mathrm{at}$ initial test (the Never-infected subcohort), follow-up time was similarly defined as time from date of initial test to the midpoint of the interval between the date of the last negative test and date of first positive PCR test. If no positive PCR test was ever recorded, followup was censored at date of the last negative PCR test. Because a PCR test is normally only conducted if the result of the Ab test is positive, we considered an $\mathrm{Ab}$ - result to be a proxy for PCR-. 'New infection' was defined as such if a member of the Never-infected cohort tested PCR+ at least once during follow-up. Confidence intervals for rates were computed using the exact Poisson method. Relative risks were estimated using Poisson regression as rate ratios adjusted for sex, age, and calendar period of test. All statistical analyses were carried out using $\mathrm{R}$ version 2.7.2 ( $\mathrm{R}$ Development Core Team, 2008).

Sensitivity analyses were conducted to assess the influence of constraining the maximum inter-test interval on the adjusted rate ratio for reinfection of previously-infected compared with infection of Never-infected subcohorts. In this analysis, we successively re-defined the study population to include only those individuals with a maximum time from initial test to first subsequent PCR test of 1 through 5 years (in steps of 0.5 years), and re-ran the regression analysis to compare the adjusted rate ratios to that obtained with no constraint on this interval (the default analysis). An additional sensitivity analysis addressed the potential influence from improvements in PCR test sensitivity over the study period; in this analysis data were restricted to initial testing conducted within the period 2002-2007 only.

\section{Results}

Demographic characteristics of the two subcohorts at date of initial test differed in terms of mean age (Table 1); the IDUs in the Never-infected subcohort were significantly younger than in the previously-infected subcohort $(M=28.4$ years; $S D=8.2$ and $\mathrm{M}=31.7$ years; $\mathrm{SD}=6.3$, respectively; $p<0.0001$ ). The median time to first subsequent test was much longer for the Neverinfected compared with the previously-infected subcohort (1.6 and 0.4 years, respectively; $p<0.0001$ ) (Table 2 ).

Median follow-up time (in which time of (re)infection was imputed), considering date of initial test to be time-zero, was 1.4 years (IQR: $0.5-3.0$ ) and 1.2 years (IQR: $0.2-3.2$ ) for the Neverinfected and previously-infected subcohorts, respectively. Of the Never-infected subcohort, 173/829 (21\%) had a subsequent positive PCR test; the infection rate was 10 per 100 person-years $(95 \%$ CI: 9-12). Of the previously-infected subcohort, 53/347 (15\%) subsequently tested PCR positive, with a reinfection rate of 7 per 100 person-years (95\% CI: 5-9). The overall incidence (aggregating new 
Table 1

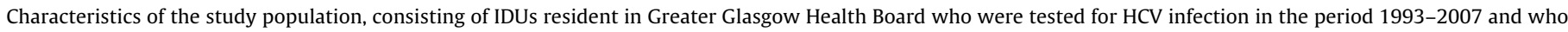
either initially tested Ab-negative (Never-infected subcohort) or initially tested Ab-positive/PCR-negative (Previously-infected subcohort).

\begin{tabular}{|c|c|c|c|}
\hline & \multicolumn{3}{|c|}{ Initial HCV test status } \\
\hline & $\mathrm{Ab}-$ & $\mathrm{Ab}+/ \mathrm{PCR}-$ & All \\
\hline Number of IDUs & 829 & 347 & 1176 \\
\hline Males (\%) & $539(65.0)$ & $216(62.2)$ & $755(64.2)$ \\
\hline Mean age at initial test, in years (SD) & $28.4(8.2)$ & $31.7(6.3)$ & $29.4(7.8)$ \\
\hline Initial test $<2000(\%)$ & $284(34.3)$ & $85(24.5)$ & $369(31.4)$ \\
\hline Initial test 2000-2003 & $393(47.4)$ & $171(49.3)$ & $564(48.0)$ \\
\hline Initial test 2004-2007 & $152(18.3)$ & $91(26.2)$ & $243(20.6)$ \\
\hline Mean number of subsequent PCR tests & 1.3 & 1.8 & 1.4 \\
\hline Median years from initial test to first subsequent PCR test (IQR) & $1.6(0.5-3.4)$ & $0.4(0.1-1.7)$ & $1.2(0.3-3.1)$ \\
\hline
\end{tabular}

Note: IQR = interquartile range; IDU = injecting drug user. IDU status was defined using risk activities fields in the HCV test and linked HIV test databases.

infections and reinfections) was 9 per 100 person-years (95\% CI: 8-11).

There was weak evidence for a reduced risk of reinfection for IDUs testing $A b+/ P C R-$ at initial test compared with those testing $\mathrm{Ab}$ - after adjustment for sex, age, and calendar period (adjusted rate ratio $[R R]=0.78,95 \% \mathrm{CI}$ : 0.57-1.08) (Table 3 ). There were statistically significant effects of age at initial test (compared with <25 years, $25-34$ years: $\mathrm{RR}=0.64,95 \% \mathrm{CI}$ : $0.48-0.85$; and $35+$ years: $\mathrm{RR}=0.56,95 \% \mathrm{CI}: 0.35-0.90$ ), and of calendar period of test (compared with 2000-2003, <2000: RR=0.55, 95\% CI: 0.37-0.82; <2004-2007: RR=0.60, 95\% CI: 0.43-0.83).

The sensitivity analysis indicated that the adjusted rate ratio for initial test status was not underestimated by including the full range of inter-test intervals; defining the eligible study population with respect to a range ( $1-5$ years) of maximum inter-test intervals resulted in adjusted rate ratios generally lower to that obtained with no inter-test interval restriction (RR central estimate ranged from 0.54 to 0.74 ). The second sensitivity analysis - addressing any effect from improvements in PCR test sensitivity over the study period - indicated a comparable estimated relative risk for initial test status ( $R R=0.82,95 \% \mathrm{CI}: 0.55-1.22)$ was obtained.

\section{Discussion}

We found only weak evidence for a reduced risk of HCV (re)infection in IDUs who had cleared their previous infection compared with IDUs who were $\mathrm{Ab}-$ at initial test. Adjustment for age (a proxy variable for risky injecting practices and injecting career length (Golub et al., 2007; Lum, Sears, \& Guydish, 2005)) and adjustment for sex and calendar period changed the unadjusted rate ratio for initial test status only slightly.

Our principal finding, though not statistically significant, is consistent in direction with results of two other cohort studies that found IDUs who had cleared infection had a significantly lower risk of (re)infection than never-infected IDUs (Grebely et al., 2006; Mehta et al., 2002). Mehta et al. reported a lower incidence of acute infection (6.0/100 person-years; $n=98)$ in previously-infected compared with never-infected IDUs (10.5/100 person-years; $n=164$ ) (Mehta et al., 2002). The other communitybased cohort study, by Grebely et al. consisted of 152 individuals (48\% of whom were self-reported IDUs) who had cleared HCV and 926 individuals (26\% IDUs) who had never been infected; the observed incidence rates of reinfection and new infection (defined as $\mathrm{Ab}$ - becoming $\mathrm{Ab+}$ ) were $1.8 / 100$ and $8.1 / 100$ person-years, respectively (Grebely et al., 2006). Our findings are inconsistent with more recent studies involving smaller cohorts of IDUs reporting a higher risk for the previously-infected group: one study reported a statistically significant adjusted rate ratio of 2.5 (Aitken et al., 2008), and the second a nonsignificant 1.1 (Micallef et al., 2007). Possible reasons for discrepant findings across studies include differences in the selection and characteristics of the two subgroups at study entry, changes in risk-taking behaviour following diagnosis with HCV infection, differences in the interval between initial and subsequent tests, and whether transient or persistent HCV infection was being measured (Currie et al., 2008).

The estimated overall HCV RNA positivity rate for this Glasgow IDU population was $9 / 100$ person-years for the period 1993-2007, lower than the seroconversion rate (28.2/100 personyears) obtained in a study involving unlinked anonymous testing of sera from Glasgow IDUs who underwent voluntary named HIV testing in the period 1993-1998 (Roy et al., 2001), but comparable to the estimate of $12 / 100$ person-years from a study of 1115 HCV Ab- IDUs attending needle-exchange services in 2008-2009 (University of the West of Scotland, Health Protection Scotland and West of Scotland Specialist Virology Centre, 2010). We also calculated a comparable incidence measure (that is, the rate of testing

Table 2

Rates of infection or reinfection in IDUs who have never been infected and IDUs who have been previously-infected with HCV, respectively.

\begin{tabular}{|c|c|c|c|c|c|c|c|}
\hline & \multicolumn{3}{|c|}{ Never-infected $(n=829)$} & \multicolumn{3}{|c|}{ Previously-infected ( $n=347$ ) } & \multirow{2}{*}{$\begin{array}{l}\text { Total } \\
\text { Rate }(95 \% \mathrm{CI})\end{array}$} \\
\hline & $\mathrm{PCR}+(\%)$ & P-yrs & Rate (95\% CI) & $\mathrm{PCR}+(\%)$ & P-yrs & Rate (95\% CI) & \\
\hline (All) & $173(20.9)$ & 1674 & $10.3(8.9-12.0)$ & $53(15.3)$ & 766 & $6.9(5.2-9.0)$ & $9.3(8.1-10.6)$ \\
\hline \multicolumn{8}{|l|}{ Sex } \\
\hline Male & 105 (19.5) & 1035 & $10.1(8.3-12.3)$ & $31(14.4)$ & 444 & $7.0(4.7-9.9)$ & $9.2(7.7-10.8)$ \\
\hline Female & $68(23.4)$ & 640 & $10.6(8.3-13.5)$ & $22(16.8)$ & 323 & $6.8(4.3-10.3)$ & $9.4(7.5-11.5)$ \\
\hline \multicolumn{8}{|c|}{ Age at initial test (years) } \\
\hline$<25$ & $93(29.6)$ & 680 & $13.7(11.0-16.7)$ & $10(18.9)$ & 110 & $9.1(4.4-16.8)$ & $13.0(10.6-15.8)$ \\
\hline $25-34$ & $72(18.6)$ & 798 & $9.0(7.1-13.4)$ & $28(14.1)$ & 499 & $5.6(3.7-8.1)$ & $7.7(6.3-9.4)$ \\
\hline $35+$ & $8(6.3)$ & 196 & $4.1(1.8-8.0)$ & $15(15.6)$ & 157 & $9.5(5.3-15.7)$ & $6.5(4.1-9.8)$ \\
\hline \multicolumn{8}{|c|}{ Calendar year period (time-dependent) } \\
\hline$<2000$ & $20(7.0)$ & 305 & $6.6(4.0-10.1)$ & $8(8.9)$ & 116 & $6.9(3.0-13.6)$ & $6.7(4.4-9.6)$ \\
\hline $2000-3$ & $122(21.0)$ & 875 & $13.9(11.6-16.7)$ & $26(10.7)$ & 376 & $6.9(4.5-10.1)$ & $11.8(10.0-13.9)$ \\
\hline $2004-7$ & $31(8.6)$ & 494 & $6.3(4.3-8.9)$ & $19(9.2)$ & 275 & $6.9(4.2-10.8)$ & $6.5(4.8-8.6)$ \\
\hline
\end{tabular}

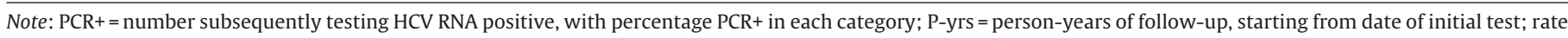
is per 100 person-years; $\mathrm{CI}=$ confidence intervals. 
Table 3

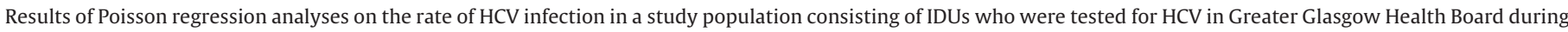

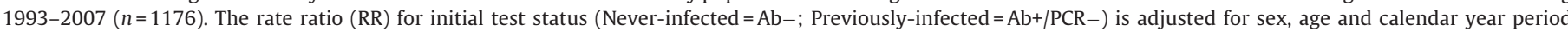
(time-dependent).

\begin{tabular}{|c|c|c|c|c|c|c|c|}
\hline Factor & Level & $N$ & $\mathrm{PCR}+$ & Unadj. RR & $95 \% \mathrm{CI}$ & Adj. RR & $95 \% \mathrm{CI}$ \\
\hline Initial test & Never-infected & 829 & 173 & Ref. & & Ref. & \\
\hline Status & Previously-infected & 347 & 53 & 0.67 & $0.49-0.91$ & 0.78 & $0.57-1.08$ \\
\hline \multirow[t]{2}{*}{ Sex } & Female & 421 & 90 & Ref. & & Ref. & \\
\hline & Male & 755 & 136 & 0.98 & $0.75-1.28$ & 1.05 & $0.80-1.37$ \\
\hline \multirow[t]{3}{*}{ Age at initial test } & $<25$ & 367 & 103 & Ref. & & Ref. & \\
\hline & $25-34$ & 586 & 100 & 0.59 & $0.45-0.78$ & 0.64 & $0.48-0.85$ \\
\hline & $35+$ & 223 & 23 & 0.50 & $0.32-0.78$ & 0.56 & $0.35-0.90$ \\
\hline \multirow[t]{3}{*}{ Calendar year period } & $<2000$ & 369 & 28 & 0.56 & $0.38-0.84$ & 0.55 & $0.37-0.82$ \\
\hline & 2000-2003 & 813 & 148 & Ref. & & Ref. & \\
\hline & 2004-2007 & 554 & 50 & 0.55 & $0.40-0.76$ & 0.60 & $0.43-0.83$ \\
\hline
\end{tabular}

Note: $N=$ number of IDUs contributing to each category; PCR+= number of IDUs subsequently testing HCV RNA positive; CI= confidence interval.

Ab and/or RNA positive) for the Never-infected group in our study, and arrived at 11/100 person-years.

Incidence increased from 7/100 person-years in 1993-1999 to $12 / 100$ person-years in 2000-2003 and then decreased to $7 / 100$ person-years in the most recent calendar period (2004-2007); the decrease between 2000-2003 and 2004-2007 may reflect trends in the rate of transmission of the virus (Hutchinson, McIntyre, \& Molyneaux, 2002), or a risk-behaviour selection effect, whereby of all IDUs initially tested during 2000-2003, those with the riskiest injection practices tended to get (re)infected relatively quickly, and those with the least risky injection practices tended to remain (re)infection-free. The decrease observed in the latter period is consistent with a downward trend in the proportion of IDUs reporting the sharing of injecting equipment (Health Protection Agency, 2011). In addition, the second test for IDUs initially tested in the latter part of the study period may not yet have occurred by the end of 2007, so these IDUs would have been excluded when determining the study population.

Our database study had several limitations. First, because IDUs were referred for subsequent HCV testing, estimated incidence rates may not be representative of those of the IDU population as a whole, because referral may have been prompted by report of risktaking behaviour. However, because members of both subcohorts were tested subsequent to index test, incidence rate ratios should be unaffected by referral bias.

We reported as much demographic data as we had available to compare the baseline characteristics of the Never-infected and previously-infected subcohorts. Because the latter group was older at study entry (by an average of 3.4 years), these individuals may engage in fewer risk-taking behaviours (for example, sharing of injecting equipment), especially after initially being diagnosed $\mathrm{HCV}$ $\mathrm{Ab}+$. Reasons for HCV test referral may differ with age, with older IDUs being referred because of a suspected higher risk of HCV infection (perhaps due to a longer reported injecting career). Further, unmeasured baseline characteristics may differ between subcohorts and account for differences in HCV (re)infection incidence.

We assumed individuals to have been at risk of acquiring HCV infection through continued injecting drug use during follow-up, but the HCV test database lacks information on ongoing injecting practice. However, age serves as a proxy variable for risk-taking behaviours - albeit far from perfect - with younger IDUs tending to engage in riskier injecting practices (Golub et al., 2007). Adjustment for age in the regression analysis should have provided some degree of control for age-related risk behaviour in the estimation of relative risk.

In an ideal prospective study addressing the current research question, a cohort of IDUs would be followed up at regular intervals from their injection debut, and a time-dependent status variable would be used to distinguish follow-up time (and therefore risk of (re)-infection) before and after initial infection; thus; individuals who clear the virus would contribute both Unexposed (pre-initial infection) and Exposed (post-initial infection) follow-up time to the analysis. However, given the necessarily retrospective design of our database-linkage study, we had to define entry to study as the initial test date and so could not address potential bias due to any unmeasured difference in Unexposed follow-up time between Never-infected and previously-infected subcohorts.

The longer interval from date of study entry to date of first $\mathrm{Ab} / \mathrm{PCR}$ test for the Never-infected subcohort means that episodes of transient viraemia were more likely to be missed in this subcohort than in the previously-infected subcohort. The presence of such bias means the rate for initially Ab-IDUs would tend to be underestimated, leading to a higher rate ratio than in reality. Related to this point, if individuals who clear HCV spontaneously are more likely to clear it again if reinfected (Osburn et al., 2010), there would be a greater chance of missing short-lived periods of viraemia in the previously-infected compared with the Neverinfected subcohort. However, the sensitivity analysis indicated only small changes in relative risk if the maximum inter-test interval was reduced substantially. Also because of the long inter-test interval (and with no subsequent test available), we could not distinguish whether having been previously-infected had an effect on reinfection risk or on viral persistence (Mehta et al., 2002).

It should be noted that the Never-infected group may include IDUs who have cleared the virus and subsequently their antibody. However, although there is no laboratory marker to detect this group, an immune recognition study of 26 young seronegative and aviremic active IDUs found that $46 \%$ had HCV-specific, IFN- $\gamma$ T-cell responses, indicating previous exposure to HCV (Zeremski et al., 2009).

Finally, reinfection rates with a different genotype may differ from rates of reinfection with same genotype. Due to insufficient data on genotype, we were unable to address this important issue. Genetic factors such as presence of an IL28B polymorphism have been shown to be associated with natural clearance of HCV (Thomas et al., 2009); we could not control for such factors.

One suggestion for the mechanism by which partial protective immunity is gained after clearing initial HCV infection centres on the deployment of adaptive immunity. In a prospective study of 22 active IDUs who had spontaneously cleared their initial HCV infection, 11 (50\%) became reinfected, but of these individuals, 83\% subsequently cleared their infection (Rahman et al., 2004). Viraemia (maximum level of HCV RNA concentration and duration) during reinfection episodes was significantly decreased compared with measurements made after initial infection in the same individuals, and a higher frequency of CD8+ T-cell responses were detected in reinfected persons, indicating that cell-mediated immune responses assist in the protective immunity resulting after clearance of initial infection. A similar pattern of reinfection and reclearance was reported in a study of 135 acutely 
infected young IDUs (Page et al., 2009). Although the immune functions underlying the responsiveness of chronic infection to combination (pegylated interferon + ribavirin) antiviral therapy are incompletely understood (Bowen \& Walker, 2005; Cramp et al., 2000), weaker HCV-specific adaptive immune responses have been observed in treatment responders compared with those who spontaneously resolved infection (Rahman et al., 2004).

Unfortunately, because our study population consists of those IDUs referred for HCV testing, being tested may play a role in terms of inducing behavioural changes, meaning that one can not distinguish the role of the test from the role of immune mechanisms in the estimated risk of reinfection reported here.

In conclusion, our findings are relevant for the goal of increasing the annual numbers of recipients of antiviral therapy proposed in the Hepatitis Action Plan for Scotland (Scottish Government Health Department, 2008). Although ongoing injecting drug use has been in practice a barrier for commencing an individual on treatment, if a reduced risk of reinfection after spontaneous viral clearance can be observed - and if also seen in those who have cleared infection through antiviral therapy (Backmund, Meyer, \& Edlin, 2004; Dalgard, 2005; Grebely et al., 2010) - this suggests that treatment of both former and current IDUs may be viable. The likelihood of reinfection should be considered when conducting an assessment of the benefits and drawbacks of antiviral therapy of this population for HCV infection.

\section{Acknowledgements}

We thank data entry staff for their support with the HCV test and HIV test databases, and the laboratories for providing the HCV and HIV test data. Funding was provided by a grant from the Chief's Scientist Office. The HCV and HIV databases were funded by the Scottish Government. The funding sources had no role in the study design; in the collection, analysis and interpretation of data; in the writing of the report; or in the decision to submit the article for publication.

\section{References}

Aitken, C. K., Tracy, S. L., Revill, P., Bharadwaj, M., Bowden, D. S., Winter, R. J., et al. (2008). Consecutive infections and clearances of different hepatitis $C$ virus genotypes in an injecting drug user. Journal of Clinical Virology, 41(4), 293-296.

Alter, M. J., Kruszon-Moran, D., Nainan, O. V., McQuillan, G. M., Gao, F., Moyer, L. A., et al. (1999). The prevalence of hepatitis C virus infection in the United States, 1988 through 1994. New England Journal of Medicine, 341(8), 556-562.

Backmund, M., Meyer, K., \& Edlin, B. R. (2004). Infrequent reinfection after successful treatment for hepatitis $C$ virus infection in injection drug users. Clinical Infectious Diseases, 39(10), 1540-1543.

Bowen, D. G., \& Walker, C. M. (2005). Adaptive immune responses in acute and chronic hepatitis $C$ virus infection. Nature, 436(7053), 946-952.

Corson, S., Greenhalgh, D., Palmateer, N., Weir, A., \& Hutchinson, S. (2011). Risk of hepatitis $C$ virus re-infection following spontaneous viral clearance in injecting drug users: A systematic review. The International Journal on Drug Policy, 22(2), $102-108$.

Cramp, M. E., Rossol, S., Chokshi, S., Carucci, P., Williams, R., \& Naoumov, N. V. (2000). Hepatitis C virus-specific T-cell reactivity during interferon and ribavirin treatment in chronic hepatitis C. Gastroenterology, 118(2), 346-355.

Currie, S. L., Ryan, J. C., Tracy, D., Wright, T. L., George, S., McQuaid, R., et al. (2008). A prospective study to examine persistent HCV reinfection in injection drug users who have previously cleared the virus. Drug and Alcohol Dependence, 93(1-2), 148-154.
Dalgard, O. (2005). Follow-up studies of treatment for hepatitis C virus infection among injection drug users. Clinical Infectious Diseases, 40(Suppl. 5), S336-S338. Dore, G. J., MacDonald, M., Law, M. G., \& Kaldor, J. M. (2003). Epidemiology of hepatitis C virus infection in Australia. Australian Family Physician, 32(10), 796-798.

Farci, P., Alter, H. J., Govindarajan, S., Wong, D. C., Engle, R., Lesniewski, R. R., et al. (1992). Lack of protective immunity against reinfection with hepatitis $C$ virus. Science, 258(5079), 135-140.

Goldberg, D., Davis, B., Allardice, G., McMenamin, J., \& Codere, G. (1996). Monitoring the spread of HIV and AIDS in Scotland 1983-1994. Scottish Medical Journal, 41(5), 131-138.

Golub, E. T., Strathdee, S. A., Bailey, S. L., Hagan, H., Latka, M. H., Hudson, S. M., et al. (2007). Distributive syringe sharing among young adult injection drug users in five U.S. cities. Drug and Alcohol Dependence, 91(Suppl. 1), S30-S38.

Grebely, J., Conway, B., Raffa, J. D., Lai, C., Krajden, M., \& Tyndall, M. W. (2006). Hepatitis $C$ virus reinfection in injection drug users. Hepatology, 44(5), 1139-1145.

Grebely, J., Knight, E., Ngai, T., Genoway, K. A., Raffa, J. D., Storms, M., et al. (2010). Reinfection with hepatitis C virus following sustained virological response in injection drug users. Journal of Gastroenterology and Hepatology, 25(7), $1281-1284$

Hay, G., Gannon, M., Casey, J., \& McKeganey, N. (2009). Estimating the national and local prevalence of problem drug misuse in Scotland: Executive report. Glasgow: University of Glasgow.

Health Protection Agency (2009). Hepatitis C in UK: Annual report 2009.

Health Protection Agency. (2011). Hepatitis C in UK 2011. London: Health Protection Agency.

Hutchinson, S., McIntyre, P., \& Molyneaux, P. (2002). Prevalence of hepatitis C among injectors in Scotland 1989-2000: Declining trends among young injectors halt in the late 1990s. Epidemiology and Infection, 128(3), 473-477.

Lum, P. J., Sears, C., \& Guydish, J. (2005). Injection risk behavior among women syringe exchangers in San Francisco. Substance Use E Misuse, 40(11), 1681-1696.

Mehta, S. H., Cox, A., Hoover, D. R., Wang, X. H., Mao, Q., Ray, S., et al. (2002). Protection against persistence of hepatitis C. Lancet, 359(9316), 1478-1483.

Micallef, J. M., Kaldor, J. M., \& Dore, G. J. (2006). Spontaneous viral clearance following acute hepatitis $C$ infection: A systematic review of longitudinal studies. Journal of Viral Hepatitis, 13(1), 34-41.

Micallef, J. M., Macdonald, V., Jauncey, M., Amin, J., Rawlinson, W., van Beek, I., et al. (2007). High incidence of hepatitis $C$ virus reinfection within a cohort of injecting drug users. Journal of Viral Hepatitis, 14(6), 413-418.

Osburn, W. O., Fisher, B. E., Dowd, K. A., Urban, G., Liu, L., Ray, S. C., et al. (2010). Spontaneous control of primary hepatitis $C$ virus infection and immunity against persistent reinfection. Gastroenterology, 138(1), 315-324

Page, K., Hahn, J. A., Evans, J., Shiboski, S., Lum, P., Delwart, E., et al. (2009). Acute hepatitis $C$ virus infection in young adult injection drug users: A prospective study of incident infection, resolution, and reinfection. Journal of Infectious Diseases, 200(8), 1216-1226.

R Development Core Team. (2008). R: A language and environment for statistical computing. Vienna; Austria: R Foundation for Statistical Computing.

Rahman, F., Heller, T., Sobao, Y., Mizukoshi, E., Nascimbeni, M., Alter, H., et al. (2004). Effects of antiviral therapy on the cellular immune response in acute hepatitis C. Hepatology, 40(1), 87-97.

Roy, K. M., Goldberg, D., Taylor, A., Hutchinson, S., MacDonald, L., Wilson, K. S., et al. (2001). A method to detect the incidence of hepatitis $C$ infection among injecting drug users in Glasgow 1993-98. Journal of Infection, 43(3), 200-205.

Scottish Government. (2011). The sexual health and blood borne virus framework, 2011-15. Edinburgh: Scottish Government.

Scottish Government Health Department. (2008). Hepatitis C action plan for Scotland: Phase II: May 2008-March 2011. Edinburgh: Scottish Government.

Thomas, D. L., Thio, C. L., Martin, M. P., Qi, Y., Ge, D., O'Huigin, C., et al. (2009). Genetic variation in IL28B and spontaneous clearance of hepatitis C virus. Nature, 461(7265), 798-801.

Trepo, C., \& Pradat, P. (1999). Hepatitis C virus infection in Western Europe. Journal of Hepatology, 31(Suppl. 1), 80-83.

University of the West of Scotland Health Protection Scotland West of Scotland Specialist Virology Centre. (2010). The needle exchange surveillance initiative (NESI): Prevalence of HCV, HIV and injecting risk behaviours among injecting drug users attending needle exchanges in Scotland, 2008/2009. Glasgow: University of the West of Scotland.

van de Laar, T. J., Molenkamp, R., van den Berg, C., Schinkel, J., Beld, M. G., Prins, M., et al. (2009). Frequent HCV reinfection and superinfection in a cohort of injecting drug users in Amsterdam. Journal of Hepatology, 51(4), 667-674.

Zeremski, M., Shu, M. A., Brown, Q., Wu, Y., Des Jarlais, D. C., Busch, M. P., et al. (2009). Hepatitis $C$ virus-specific T-cell immune responses in seronegative injection drug users. Journal of Viral Hepatitis, 16(1), 10-20. 